Pacific Journal of Mathematics

TOPOLOGICAL GROUPS WHOSE UNDERLYING SPACES ARE 


\section{TOPOLOGICAL GROUPS WHOSE UNDERLYING SPACES ARE SEPARABLE FRÉCHET MANIFOLDS}

\section{JAMES KEESLING}

Let $G$ be a topological group and let $G^{*}$ denote the space of all Lebesgue measurable functions from the unit interval $[0,1]$ into $G$ with the topology of convergence in measure. With this topology and with pointwise multiplication as the group operation, $G^{*}$ is a topological group. If $G$ is separable and has a complete metric and has more than one point, then Bessaga and Pelczyński have shown that $G^{*}$ is homeomorphic to $l_{2}$, separable infinite-dimensional Hilbert space. This fact is used in this paper to show the existence of separable Fréchet manifolds which are topological groups and which have certain algebraic and topological properties.

Introduction. Let $X$ be a metric space and $X^{*}$ be the space of all Lebesgue measurable functions from $[0,1]$ into $X$ with the topology of convergence in measure. The space $X^{*}$ is a contractible, locally contractible metric space in general. If $X$ has a complete metric, then $X^{*}$ also has a complete metric. Bessaga and Pełczyński [3] have shown that if $X$ is $\{0,1\},[0,1]$, or $R$, then $X^{*}$ is homeomorphic to $l_{2}$, separable infinite-dimensional Hilbert space. More recently they have shown [4] that $X^{*}$ is homeomorphic to $l_{2}$ whenever $X$ is a complete separable metric space with more than one point. This fact is used in this paper to show the existence of topological groups which are connected separable Fréchet manifolds and which have certain algebraic and topological properties. The construction we use is a modification of one used by Hartman and Mycielski [8]. Primarily we study two types of topological groups: periodic topological groups and those topological groups whose underlying group structure is isomorphic to the additive reals. The results are contrasted with the structure theory of locally compact topological groups [10 and 16] and with the theory of infinite-dimensional Lie groups [14].

In the last section of the paper it is shown that if $\pi$ is any abelian group, then $\pi$ is the fundamental group of a completely metrizable topological group $G_{\pi}$ which is a $K(\pi, 1)$. If $\pi$ is countable, then $G_{\pi}$ is a separable Fréchet manifold. If $\pi$ is any group, then we show the existence of a completely metrizable homogeneous space which is a $K(\pi, 1)$. If $\pi$ is countable, then the space will be 
a separable Fréchet manifold. F. E. A. Johnson [12] has shown that for (finite-dimensional) manifolds if $\pi$ is a group, then there is a manifold $M^{n}$ of type $K(\pi, 1)$ if and only if $\pi$ is countable and has finite cohomological dimension. This contrasts with infinite-dimensional manifolds which are of type $K(\pi, 1)$. Milnor [17] has shown that if $\pi$ is a countable abelian group and $n$ is any positive integer, then there is a topological group which is a $K(\pi, n)$, but the space is not a separable Fréchet manifold. The author wishes to thank the referee for referring him to the papers of Milnor and Johnson.

Notation. If $X$ and $Y$ are topological spaces, then $X \sim Y$ will mean that $X$ and $Y$ are homeomorphic. We let $R$ denote the real numbers with the usual topology. We consider $R$ as a topological group with addition as the operation. The rational numbers are denoted by $Q$, the integers by $Z$, and the set $\{0,1,2, \cdots, n-1\}$ by $Z_{n}$. The sets $Z$ and $Z_{n}$ have the discrete topology and are considered to be groups with the usual addition. Separable infinite-dimensional Hilbert space is denoted by $l_{2}$. A separable Fréchet manifold is a separable metric space $X$ such that for each $x \in X$, there is an open set $U_{x}$ in $X$ containing $x$ such that $U_{x} \sim l_{2}$. This terminology comes from the fact that all separable infinite-dimensional Fréchet spaces are homeomorphic. See the Introduction in [1] for a list of references to results which, taken together, prove this. If $X$ is a topological space, then $\pi_{n}(X)=\pi_{n}(X, x)$ denotes the $n$th homotopy group of $X$ with base point $x$.

We assume the rudiments of measure theory as contained in [18]. We let $\lambda$ denote Lebesgue measure on $[0,1]$. If $f:[0,1] \rightarrow R$ is Lebesgue integrable, then the integral of $f$ is denoted $\int f d \lambda$.

1. The space of measurable functions. Let $X$ be a metric space with bounded metric $d$. Let $X^{*}$ be the set of all Lebesgue measurable functions from $[0,1]$ into $X$. We identify two functions if the set on which they differ has measure zero. Define $\rho_{d}(f, g)=$ $\int d(f(t), g(t)) d \lambda$. Then $\rho_{d}$ is a bounded metric on $X^{*}$. If $\left\{f_{i}\right\}_{i=1}^{\infty}$ is a sequence of measurable functions $f_{i}:[0,1] \rightarrow X$, then $\left\{f_{i}\right\}_{i=1}^{\infty}$ converges in measure to the measurable function $f:[0,1] \rightarrow X$ provided that $\lambda\left(\left\{t \in[0,1]: d\left(f_{i}(t), f(t)\right) \geqq \varepsilon\right\}\right)=\delta_{i} \rightarrow 0$. It should be clear that $f_{i}$ converges in measure to $f$ if and only if $\rho_{d}\left(f_{i}, f\right) \rightarrow 0$. Thus the topology induced on $X^{*}$ by $\rho_{d}$ is the same as that induced by $\rho_{\hat{o}}$ where $\delta$ is any other bounded metric inducing the topology of $X$. The space $X^{*}$ with the topology induced by $\rho_{d}$ is called the space of all Lebesgue measurable functions with the topology of convergence 
in measure.

If $X$ is a topological group or a topological vector space, then $X^{*}$ is also with the operations induced by the pointwise operations. Certain spaces $X^{*}$ are important in analysis. The space $\{0,1\}^{*}$ is isomorphic to the measure algebra of $[0,1]$ and $R^{*}$ is the standard example of a complete separable metric topological vector space which is not locally convex. Recently Bessaga and Pełczyński [3] have shown that $\{0,1\}^{*},[0,1]^{*}$, and $R^{*}$ are all homeomorphic to $l_{2}$. More recently they have shown the following [4].

Theorem 1.1. (Bessaga and Pełczyński): Let $X$ be any complete separable metric space with more than one point. Then $X^{*} \sim l_{2}$.

This characterizes those spaces $X$ for which $X^{*}$ is homeomorphic to $l_{2}$. In particular, if $X$ is a countable discrete space with more than one point, then $X^{*}$ is homeomorphic to $l_{2}$.

As pointed out in [4], Theorem 1.1 together with the remarks above and results in [19] implies that: (1) every separable metric topological group can be imbedded as a subgroup of a topological group homeomorphic to $l_{2} ;$ (2) every complete separable metric topological group can be imbedded as a closed subgroup of a topological group homeomorphic to $l_{2}$; (3) every separable metric topological group which contains at least two points has a free action on $l_{2}$; and (4) if $G$ is a separable metric topological group with at least two points and $F$ is any closed subset of $l_{2}$, there is a semifree action of $G$ on $l_{2}$ with $F$ as its set of fixed points. In [19] (4) was shown for the case that $G$ was compact.

In this paper Theorem 1.1 will be used to give simple examples of topological groups whose underlying spaces are separable Fréchet manifolds and which in addition have certain algebraic and topological properties as well.

2. Periodic topological groups. A group $G$ is said to be periodic provided that each element of $G$ has finite order. It is said to be of period $n$ provided that each element of $G$ has order at most $n$ and some element has order $n$. By a very complicated means Markov [15] showed that there exist connected periodic topological groups of period two. Hartman and Mycielski [8] pointed out that $\{0,1\}^{*}$ is an easier example of this. They also pointed out that for each positive integer $n$, there are arcwise connected, locally arcwise connected, metrizable topological groups $G_{n}$ which are periodic of period $n$. Here we use Theorem 1.1 to show that if $H$ is any countable periodic group of period $n$, then there is a topological 
group $G_{H}$ which is a separable Fréchet manifold whose fundamental group is $H$. It should be pointed out that if $G$ is a locally compact group which is not totally disconnected, then $G$ contains a nondegenerate one-parameter subgroup (see [16, Theorem 1, p. 192]). Consequently, such a group could not be periodic.

THEOREM 2.1. If $G_{n}$ is a periodic topological group of period at most $n$, then for each positive integer $k, \pi_{k}\left(G_{n}\right)$ is a periodic group of period at most $n$.

Proof. Let $\Omega\left(G_{n}\right)$ denote the loop space of $G_{n}$ with base point $1 \in G_{n}$ and let $\Omega_{0}\left(G_{n}\right)$ denote the contractible loops. Let $m$ denote pointwise multiplication of loops in $\Omega\left(G_{n}\right)$. With the compact open topology on $\Omega\left(G_{n}\right), m$ is homotopic to the ordinary loop multiplication in $\Omega\left(G_{n}\right)$ and thus $\pi_{1}\left(G_{n}\right) \cong \Omega\left(G_{n}\right) / \Omega_{0}\left(G_{n}\right)$ is periodic of period at most $n$. For the higher homotopy groups the result follows by induction using the fact that $\pi_{k+1}\left(G_{n}\right) \cong \pi_{k}\left(\Omega_{0}\left(G_{n}\right)\right)[11, \mathrm{p} .109]$ and the fact that $\Omega_{0}\left(G_{n}\right)$ is a topological group which is periodic of period at most $n$ under the multiplication $m$.

ExAmple 2.2. Let $P^{3}$ be real projective 3 -space. Then $P^{3}$ is a Lie group and $P^{3} \times l_{2}$ is a separable Fréchet manifold which is a topological group. The fundamental group of $P^{3} \times l_{2}$ is just $Z_{2}$ which is periodic of period two. However, $P^{3} \times l_{2}$ does not admit a topological group structure which is periodic of period two. This is because $\pi_{3}\left(P^{3} \times l_{2}\right)=\pi_{3}\left(P^{3}\right)=\pi_{3}\left(S^{3}\right)=Z$ is not periodic of period two.

THEOREM 2.3. Let $H$ be any abelian group which is periodic of period n. Then there is a topological group $G_{H}$ which is periodic of period $n$ which is completely metrizable and locally contractible such that $\pi_{1}\left(G_{H}\right) \cong H$. If $H$ is finite or countably infinite, then $G_{H}$ is a separable Fréchet manifold.

Proof. Let $H$ be the given group and give it the discrete topology. Identify $H$ with the constant functions in $H^{*}$. Let $G_{H}=$ $H^{*} / H$ which is clearly periodic of period $n$. Then the natural map $\nu: H^{*} \rightarrow H^{*} / H$ is a covering projection with $H^{*}$ completely metrizable, contractible, and locally contractible. Thus $H^{*} / H$ is completely metrizable and locally contractible. Since $H^{*}$ is contractible,

$$
\pi_{1}\left(H^{*} / H\right) \cong \operatorname{ker} \nu=H
$$

[6, Theorem 4.5, p. 14]. If $H$ is finite or countably infinite, then $H^{*}$ is homeomorphic to $l_{2}$ by Theorem 1.1 and thus $H^{*} / H$ is a separable Fréchet manifold. 
3. Topological groups isomorphic to the additive reals. The additive group of real numbers is characterized as a vector space over the rational numbers $Q$ of cardinality $2^{\aleph_{0}}$. The principal examples of locally compact groups whose underlying group is isomorphic to the additive reals are the reals $R$ and the rational solenoid $\Sigma_{\omega}[7]$. In fact, the only locally compacted topological groups having this underlying group structure are of the form $R^{n} \times \prod_{i \in I} \Sigma_{\omega}$ where $n$ is a nonnegative integer and $2^{\text {card } I} \leqq 2^{\aleph_{0}}$ [13]. Consequently, for any locally compact topological group $G$ with $G$ group isomorphic to $R$ and any positive integer $m, \pi_{m}(G)=0$. This contrasts with Theorem 3.5 where we show that a topological group $G$ which is a separable Fréchet manifold with $G$ group isomorphic to $R$ may have nontrivial fundamental group.

Infinite-dimensional topological groups which are homeomorphic to $l_{2}$ and whose group structure is the additive reals are abundant. Any separable infinite dimensional Banach space is an example of one. These examples are Lie groups in the sense of Maissen [14]. The Fréchet space $R^{\infty}$ is an example of one which is not a Lie group in the sense of [14]. Our next theorems show that there may be a much more complicated relationship between the algebraic structure of an infinite-dimensional topological group and its topological structure than these examples would indicate.

THEOREM 3.1. There is a topological group $G$ whose underlying group is isomorphic to the additive reals with $G \sim l_{2}$ such that $G$ contains no nontrivial continuous one-parameter subgroups.

Proof. Let $H$ be any countable vector space over the rationals $Q$ and let $H$ have the discrete topology with metric $d(x, y)=1$ for all $x \neq y$. Let $G=H^{*}$. Then $G \sim l_{2}$ by Theorem 1.1. Clearly $G$ is a topological group whose underlying group structure is a vector space over $Q$ with cardinality $2^{\aleph_{0}}$. Now suppose that $\phi: R \rightarrow G$ is a continuous one-parameter subgroup in $G$. Suppose that $\phi(x)=g \neq 0$ in $G$. Now $x / n \rightarrow 0$. Therefore $\phi(x / n)=g / n \rightarrow 0$. Now $g:[0,1] \rightarrow H$ has the property that $\rho_{d}(g, 0)=\int d(g(t), 0) d \lambda=\int d((g(t)) / n, 0) d \lambda=\rho_{d}(g / n, 0)$ for all $n$. Thus $\rho_{d}(g / n, 0) \nrightarrow 0$ and $g / n \nrightarrow 0$, a contradiction. Therefore $\phi$ must be identically zero and $G$ has no nontrivial continuous oneparameter subgroups.

Theorem 3.1 should be contrasted with the fact that every locally compact topological group which is not zero-dimensional has a nondegenerate one-parameter subgroup as mentioned earlier. This is also true of infinite-dimensional Lie groups [14].

Let $G$ be a group and $n$ a positive integer. Then $G$ is said to 
be $n$-divisible provided that for each element $x$ in $G$, there is a $y$ in $G$ with $y^{n}=x$. A torsion free abelian group is a vector space over $Q$ if and only if it is $n$-divisible for all $n$.

The following proposition was first proved by Banach [2].

Proposition (Banach) 3.2. If $G$ and $H$ are separable and completely metrizable topological groups and if $\phi: G \rightarrow H$ is a surjective continuous homomorphism, then $\phi$ is open.

COROLlary 3.3. Let $G$ be a separable, completely metrizable topological group which is abelian, torsion free, and n-divisible. Then the mapping which takes $x$ to $x / n$ is a topological group isomorphism.

ExAmple 4.6. The following example shows that completeness is necessary in Corollary 3.3 even though a special homomorphism is involved. Let $Q$ be the rationals and let $N_{n}=\left\{k 2^{n}: k \in Z\right\}$. Then $\left\{N_{n}\right\}_{n=1}^{\infty}$ forms a basis for the neighborhoods of 0 in $Q$ in a topology $\tau$ on $Q$ which makes $Q$ into a metrizable topological group [10, p.18]. Now in the topology $\tau$, the sequence $x_{i}=2^{i}$ has the property that $x_{i} \rightarrow 0$. However, $\left\{x_{i} / 3\right\}_{i=1}^{\infty}$ does not converge to 0 in $Q$ in the topology $\tau$. Thus the map taking $x$ to $x / 3$ is not continuous even though $Q$ is 3 -divisible.

THeOREM 3.4. If $G$ is a separable completely metrizable topological group whose underlying group is a vector space over $Q$, then $\pi_{n}(G)$ is a vector space over $Q$ for every positive integer $n$.

Proof. Let $G$ be written additively. Let $\Omega(G)$ and $\Omega_{0}(G)$ be the loop space of $G$ with base point 0 and the contractible loops, respectively. Let $m$ be pointwise multiplication on $\Omega(G)$ and $\Omega_{0}(G)$. Then, as pointed out in the proof of Theorem 2.1, $\Omega(G)$ and $\Omega_{0}(G)$ are topological groups. Clearly $\Omega(G)$ is a vector space over $Q$. Let $n$ be a positive integer. Now $\Omega(G)$ is completely metrizable and separable. Therefore the map taking $g$ to $g / n$ is a topological group isomorphism. Thus division by $n$ takes $\Omega_{0}(G)$ onto itself. Thus $\Omega_{0}(G)$ is $n$-divisible for all $n$ and a vector space over $Q$. Thus the factor group $\Omega(G) / \Omega_{0}(G)$ is also a vector space over $Q$. Since $\pi_{1}(G) \cong \Omega(G) / \Omega_{0}(G)$ the theorem is proved for the case $n=1$. Now $\Omega(\Omega(G))=\Omega\left(\Omega_{0}(G)\right)$ since each loop in $\Omega(G)$ with base point 0 must be contained in the arc-component of 0 in $\Omega(G)$ which is just $\Omega_{0}(G)$. Now for $n \geqq 1$, $\pi_{n+1}(G) \cong \pi_{n}\left(\Omega\left(\Omega_{0}(G)\right)\right) \pi_{n}(\Omega(\Omega(G))$ and the theorem follows by induction. 
THEOREM 3.5. Let $H$ be any abelian group which is a countable vector space over the rationals. Then there is a topological group $G$ which is a connected separable Fréchet manifold such that $\pi_{1}(G)=H$ such that the underlying group structure of $G$ is isomorphic to the additive reals.

Proof. Let $H$ have the discrete topology. Identify $H$ with the constant functions in $H^{*}$. It is clear that $H^{*}$ is a vector space over $Q$ with subspace $H$. Therefore $H^{*} / H$ is a vector space over $Q$. Thus $G=H^{*} / H$ is the required group.

Similarly we have the next result.

THEOREM 3.6. Let $H$ be any group which is a vector space over Q. Then there is a completely metrizable topological group $G$ which is locally contractible such that the underlying group of $G$ is a vector space over $Q$ and such that $\pi_{1}(G) \cong H$.

4. Topological groups which are of type $K(\pi, 1)$. Theorem 1.1 also gives us a simple way to construct separable Fréchet manifolds which are $K(\pi, 1)$ 's for any countable group $\pi$.

THEOREM 4.1. Let $\pi$ be any abelian group. Then there is a completely metrizable, locally contractible, connected topological group $G_{\pi}$ of type $K(\pi, 1)$. If $\pi$ is countable, then $G_{\pi}$ is a separable Fréchet manifold.

Proof. Let $\pi$ be given the discrete topology and identify $\pi$ with the constant functions in $\pi^{*}$. Then $G_{\pi}=\pi^{*} / \pi$ is the required topological group.

A homogeneous space is a topological space $X$ such that $X \sim G / H$ where $G$ is a topological group, $H$ is a closed subgroup, and $G / H$ is the decomposition space of right (or left) cosets of $H$ with quotient topology. If $\pi$ is any group, then $\pi^{*} / \pi$ is a homogeneous space which is a $K(\pi, 1)$.

THEOREM 4.2. Let $\pi$ be any group, Then there is a completely metrizable, locally contractible, homogeneous space $M_{\pi}$ which is a $K$ $(\pi, 1)$. If $\pi$ is finite or countably infinite, then $M_{\pi}$ is a separable Fréchet manifold.

Note 4.3. If $X$ is any separable Fréchet manifold which is a $K(\pi, 1)$, then all homotopy groups of the covering space $\tilde{X}$ of $X$ 
vanish. Since $\tilde{X}$ is also a separable Fréchet manifold, it has the homotopy type of a $C W$-complex. Thus $\widetilde{X}$ is contractible. By [9], $\tilde{X}$ must be homeomorphic to $l_{2}$. In the examples given above this is clearly the case.

The last results in the paper have to do with the higher homotopy groups of topological groups which are separable Fréchet manifolds.

THEOREM 4.4. Let $G$ be a topological group which is a separable Fréchet manifold and suppose that $H$ is any finite or countable abelian group and that $N$ is any nonnegative integer. Then there is a topological group $G^{\prime}$ which is a separable Fréchet manifold having the property that $\pi_{1}\left(G^{\prime}\right)=H$ and $\pi_{n}\left(G^{\prime}\right)=\pi_{n+N}(G)$ for all $n \geqq 2$.

Proof. First of all suppose that $H=\{0\}$. Define

$$
\Omega_{n}(G)=\Omega\left(\Omega_{n-1}(G)\right)
$$

where $\Omega_{1}(G)=\Omega(G)$. Clearly $\Omega_{n}(G)$ is a topological group for all $n$. We will now show that $\Omega_{n}(G)$ is a separable Fréchet manifold for all $n$. If $G \sim l_{2}$, then this is true since $\Omega(G)$ can be given the structure of an infinite-dimensional Banach space. If $G$ is an arbitrary separable Fréchet manifold, then $G$ can be imbedded as an open subset of $l_{2}$ by [9]. Thus $\Omega(G)$ can be considered to be an open subset of $\Omega\left(l_{2}\right) \sim l_{2}$ and thus is a separable Fréchet manifold. By induction, $\Omega_{n}(G)$ is a separable Fréchet manifold. To complete the proof for $H=\{0\}$ simply let $G^{\prime}$ be the universal covering group of the identity component of $\Omega_{N}(G)$ if $N \geqq 1$ and the universal covering group of the identity component of $G$ if $N=0$.

If $H \neq\{0\}$, then let $G_{1}$ be the topological group just constructed for the case $H=\{0\}$ and let $G_{2}$ be the topological group $G_{H}$ in Theorem 4.1. Then let $G^{\prime}=G_{1} \times G_{2}$.

REMARK 4.5. In this remark we state some facts about Lie groups in order to contrast them with Theorems 4.1 and 4.4. First of all, if $L$ is a connected Lie group, then $L$ is homeomorphic to $R^{n} \times K$ where $K$ is a compact Lie group [16, pp. 188-189]. Thus every Lie group has the homotopy type of a compact Lie group and thus of a compact manifold. Therefore its fundamental group must be finitely presented. By Theorem 4.1, the fundamental group of a topological group which is a separable Fréchet manifold need not be finitely presented.

Another fact about Lie groups is that $\pi_{2}(L)=0$. Theorem 4.4 shows that this need not be true for topological groups which are 
separable Fréchet manifolds. To show this let $L$ be any compact simply connected Lie group. Then $\pi_{k}(L) \neq 0$ for some $k \geqq 3$. Then let $H=L \times l_{2}$ which is a topological group and a separable Fréchet manifold. Then let $G^{\prime}$ be the group given by Theorem 4.4 for $n=$ $k-2$ and $H$ arbitrary. Then $\pi_{2}\left(G^{\prime}\right)=\pi_{k}(L) \neq 0$.

\section{REFERENCES}

1. R. D. Anderson and R. H. Bing, A complete elementary proof that Hilbert space is homeomorphic to the countable infinite product of lines, Bull. Amer. Math. Soc. 74 (1968), 771-792.

2. S. Banach, Über metrische Gruppen, Studia Math. 3 (1931), 101-113.

3. C. Bessaga and A. Pelczyński, The space of Lebesgue measurable functions on the interval $[0,1]$ is homeomorphic to the countable infinite product of lines, Math. Scand. 27 (1970), 132-140.

4. - (to appear).

5. J. Dugundji, Topology, Allyn and Bacon, Boston, 1965.

6. M. Greenberg, Lectures on Algebraic Topology, Benjamin, New York, 1967.

7. P. R. Halmos, Comment on the real line, Bull. Amer. Math. Soc., 50 (1944), 877878.

8. S. Hartman and J. Mycielski, On the imbedding of topological groups into connected topological groups, Colloq. Math., 5 (1958), 167-169.

9. D. W. Henderson, Infinite-dimensional manifolds are open subsets of Hilbert space, Topology 9 (1970), 155-161.

10. E. Hewitt and K. Ross, Abstract Harmonic Analysis I, Springer-Verlag, New York, 1963.

11. S. T. Hu, Homotopy, Academic Press, New York, 1959.

12. F. E. A. Johnson, Manifolds of homotopy type $K(\pi, 1)$. I, Proc. Camb. Phil. Soc., 70 (1971), 387-393.

13. G. Mackey, A remark on locally compact abelian groups, Bull. Amer. Math. Soc. 52 (1946), 940-944.

14. B. Maissen, Lie-Gruppen mit Banachräumen als Parameterräume, Acta Math. 108 (1962), 229-270.

15. A. A. Markov, On the existence of periodic connected topological groups, (Russian) Izv. Akad. Nauk SSSR Ser. Math., 8 (1944), 225-232 = Amer. Math. Soc. Trans. (1) 8 (1962), 186-194.

16. D. Montgomery and L. Zippin, Topological Transformation Groups, John Wiley, New York, 1955.

17. J. Milnor, The geometric realization of a semi-simplicial complex, Ann. of Math., 65 (1957), 357-362.

18. H. L. Royden, Real Analysis, Macmillan, New York, 1963.

19. J. E. West, Fixed point sets of transformation groups of infinite product spaces, Proc. Amer. Math. Soc. 21 (1969), 575-582.

Received August 10, 1971 and in revised form March 20, 1972. This research was supported by National Science Foundation Grant GP-24616.

UNIVERSITY OF FLORIDA 



\section{PACIFIC JOURNAL OF MATHEMATICS}

\section{EDITORS}

H. SAMELSON

Stanford University

Stanford, California 94305

C. R. Hовву

University of Washington

Seattle, Washington 98105
J. DugundJI

Department of Mathematics University of Southern California

Los Angeles, California 90007

RICHARD ARENS

University of California

Los Angeles, California 90024

\section{ASSOCIATE EDITORS}

E. F. BECKENBACH

B. H. NeumanN

F. WOLF

K. YoSHIDA

\section{SUPPORTING INSTITUTIONS}

UNIVERSITY OF BRITISH COLUMBIA

CALIFORNIA INSTITUTE OF TECHNOLOGY

UNIVERSITY OF CALIFORNIA

MONTANA STATE UNIVERSITY

UNIVERSITY OF NEVADA

NEW MEXICO STATE UNIVERSITY

OREGON STATE UNIVERSITY

UNIVERSITY OF OREGON

OSAKA UNIVERSITY
UNIVERSITY OF SOUTHERN CALIFORNIA

STANFORD UNIVERSITY

UNIVERSITY OF TOKYO

UNIVERSITY OF UTAH

WASHINGTON STATE UNIVERSITY

UNIVERSITY OF WASHINGTON

AMERICAN MATHEMATICAL SOCIETY

NAVAL WEAPONS CENTER

Printed in Japan by International Academic Printing Co., Ltd., Tokyo, Japan 


\section{Pacific Journal of Mathematics}

\section{Vol. 44, No. $1 \quad$ May, 1973}

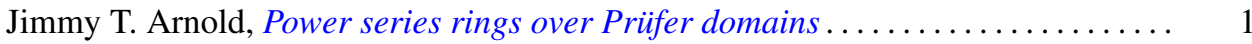

Maynard G. Arsove, On the behavior of Pincherle basis functions . . . . . . . . . 13

Jan William Auer, Fiber integration in smooth bundles ................. 33

George Bachman, Edward Beckenstein and Lawrence Narici, Function algebras

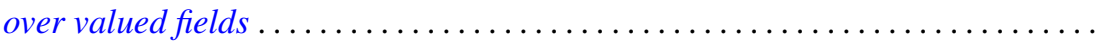

Gerald A. Beer, The index of convexity and the visibility function . . . . . . . . . . .

James Robert Boone, A note on mesocompact and sequentially mesocompact

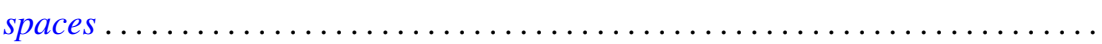

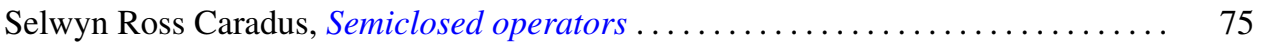

John H. E. Cohn, Two primary factor inequalities . . . . . . . . . . . . . . . 81

Mani Gagrat and Somashekhar Amrith Naimpally, Proximity approach to

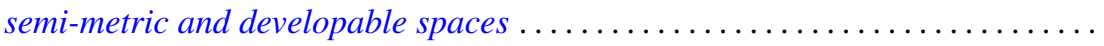

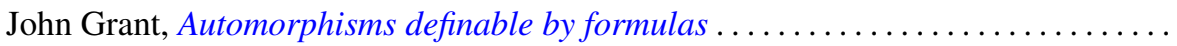

Walter Kurt Hayman, Differential inequalities and local valency ..............

Wolfgang H. Heil, Testing 3-manifolds for projective planes . . . . . . . . . . . . .

107

Melvin Hochster and Louis Jackson Ratliff, Jr., Five theorems on Macaulay

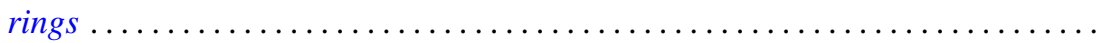

Thomas Benton Hoover, Operator algebras with reducing invariant subspaces ....

James Edgar Keesling, Topological groups whose underlying spaces are separable

Fréchet manifolds...

Frank Leroy Knowles, Idempotents in the boundary of a Lie group . .

191

George Edward Lang, The evaluation map and EHP sequences ...

201

Everette Lee May, Jr, Localizing the spectrum . . . . . . . . . . . .

211

Frank Belsley Miles, Existence of special $K$-sets in certain locally compact abelian groups.

Susan Montgomery, A generalization of a theorem of Jacobson. II . .

T. S. Motzkin and J. L. Walsh, Equilibrium of inverse-distance forces in

three-dimensions.

Arunava Mukherjea and Nicolas A. Tserpes, Invariant measures and the converse

of Haar's theorem on semitopological semigroups .

James Waring Noonan, On close-to-convex functions of order $\beta$

Donald Steven Passman, The Jacobian of a growth transformation

Dean Blackburn Priest, A mean Stieltjes type integral ........ .

Joe Bill Rhodes, Decomposition of semilattices with applications to topological

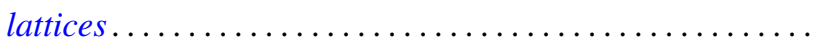

Claus M. Ringel, Socle conditions for $\mathrm{QF}-1$ rings ..........

Richard Rochberg, Linear maps of the disk algebra

Roy W. Ryden, Groups of arithmetic functions under Dirichlet convolution . .

Michael J. Sharpe, A class of operators on excessive functions

Erling Stormer, Automorphisms and equivalence in von Neumann algebras ..

Philip C. Tonne, Matrix representations for linear transformations on series

analytic in the unit disc. 